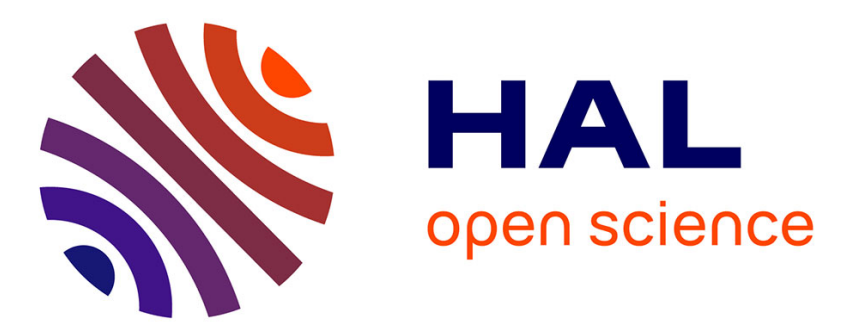

\title{
Simple methods for crosstalk reduction in fiber optical parametric amplifiers
}

\author{
Arnaud Mussot, Eric Lantz, Anne Durécu-Legrand, Christian Simmoneau, \\ Dominique Bayart, Hervé Maillotte, T. Sylvestre
}

\section{- To cite this version:}

Arnaud Mussot, Eric Lantz, Anne Durécu-Legrand, Christian Simmoneau, Dominique Bayart, et al.. Simple methods for crosstalk reduction in fiber optical parametric amplifiers. Optics Communications, 2007, 275 (2), pp.448-452. 10.1016/j.optcom.2007.03.048 . hal-00269147

\section{HAL Id: hal-00269147 https://hal.science/hal-00269147}

Submitted on 29 Aug 2013

HAL is a multi-disciplinary open access archive for the deposit and dissemination of scientific research documents, whether they are published or not. The documents may come from teaching and research institutions in France or abroad, or from public or private research centers.
L'archive ouverte pluridisciplinaire HAL, est destinée au dépôt et à la diffusion de documents scientifiques de niveau recherche, publiés ou non, émanant des établissements d'enseignement et de recherche français ou étrangers, des laboratoires publics ou privés. 


\title{
Simple methods for crosstalk reduction in fiber optical parametric amplifiers
}

\author{
A. Mussot ${ }^{\text {a,b }}$, E. Lantz ${ }^{\text {a }}$, A. Durécu-Legrand ${ }^{\text {c,1 }}$, C. Simonneau ${ }^{\text {c }}$, D. Bayart ${ }^{\text {c }}$, \\ H. Maillotte ${ }^{\text {a }}$, T. Sylvestre ${ }^{\text {a,* }}$ \\ a Département d'Optique P.M. Duffieux, Institut FEMTO-ST, UMR CNRS 6174, Université de Franche-Comté, 16 route de Gray, \\ F-25030 Besançon Cedex, France \\ ${ }^{\mathrm{b}}$ Laboratoire de Physique des Lasers Atomes et Molécules, Université des Sciences et Technologies de Lille, UFR de Physique, \\ 59655 Villeneuve d'Asq Cedex, France \\ ${ }^{\mathrm{c}}$ Alcatel, Research and Innovation Department, Route de Nozay, F-91460 Marcoussis, France
}

Received 20 November 2006; received in revised form 21 March 2007; accepted 21 March 2007

\begin{abstract}
We propose and numerically demonstrate two simple methods for reduction of the crosstalk caused by four-wave mixing between the pump and the wavelength-division multiplexed channels in single-pump fiber optical parametric amplifier. The first one is based on a proper tuning of the WDM system from the pump wavelength and the second one uses the Stokes side rather than the anti-Stokes side in the gain band.
\end{abstract}

(c) 2007 Elsevier B.V. All rights reserved.

Keywords: Crosstalk; Fiber optical parametric amplifier (FOPA); Wavelength-division multiplexing (WDM)

\section{Introduction}

Crosstalk due to four-wave mixing (FWM) in fiber optical parametric amplifiers (FOPA) is a crucial issue for wavelength-division multiplexing (WDM) systems. It has been recently identified experimentally as a main cause of system degradation and power penalty in FOPA driven by one or two-pump waves [1-7]. In both cases, the origin of FWM-induced crosstalk is twofold. First, unwanted frequencies arising from channel-channel parametric FWM (CC) are efficiently generated because of the low group-velocity dispersion and high nonlinearity of the fiber used in FOPAs [1-6]. Second, there is an additional source of crosstalk resulting from strong pump-channel

\footnotetext{
${ }^{*}$ Corresponding author. Tel.: +33 381666425; fax: +33381666423

E-mail addresses: mussot@phlam.univ-lille1.fr (A. Mussot), thibaut. sylvestre@univ-fcomte.fr (T. Sylvestre).

${ }^{1}$ Present address: ONERA DOTA/SLS Chemin de la Hunière, F-91761 Palaiseau Cedex.
}

(PC) parametric FWM interactions [7]. The impact of this crosstalk on system degradation has already been identified in the framework of single-pump fiber parametric amplifiers for wavelength band conversion in a multi-span configuration [8]. However, it has not been yet considered in detail with realistic intensity modulated signals in the case of a single-span FOPA configuration. The purpose of this work is to show, by a numerical analysis, that this PC crosstalk can be significant with respect to classical $\mathrm{CC}$ crosstalk and that it can be easily reduced by properly tuning the WDM system allocation with respect to the pump frequency. In addition, we point out that the overall crosstalk is less important on the Stokes side of the FOPA.

This paper is organized as follows: in Section 2 we investigate the PC crosstalk through simple phase-matching considerations which are verified in Section 3 by numerical simulations of a realistic WDM system amplified by a single-pump fiber optical parametric amplifier. We then discuss our results in Section 4 and give our conclusions. 


\section{Pump-channel crosstalk}

In FOPAs, WDM channels are generally located near the pump frequency to benefit from the whole gain band. Consequently, in case of WDM signals, there exists a PC crosstalk which results from FWM interaction involving the high-power pump wave [7]. These interactions are schematically sketched in Fig. 1. We consider here an equallyspaced WDM system made up of $N$ signals propagating in a single-pump FOPA. The channel spacing and the frequency detuning between the first channel and the pump are denoted $\Delta \omega$ and $\Delta \Omega$, respectively. Let us consider the FWM interaction between the pump, the $n$th channel and the $(n-1)$ th one that leads to the generation of a parasitic wave located at $\omega_{\mathrm{p}}^{+}$according to energy conservation relation: $\omega_{\mathrm{p}}+\omega_{n}=\omega_{n}^{-}+\omega_{\mathrm{p}}^{+}$. It can be generalized to the interaction between the pump, the $n$th channel and the $(n \pm k)$ th channel which creates a crosstalk located at $\omega_{\mathrm{P}} \pm k \times \Delta \omega$ by the relation

$\omega_{\mathrm{p}}+(\Delta \Omega+n \Delta \omega)=\left(\omega_{\mathrm{p}} \pm k \times \Delta \omega\right)+(\Delta \Omega+(n \mp k) \times \Delta \omega)$

It is worth noting that parasitic waves whose frequencies correspond to the first term of the second member of Eq. (1) are always spaced from the pump by $k$ times the channel-channel spacing $(\Delta \omega)$, with $k$ an integer. Under these conditions, parasitic frequencies arising from the whole PC FWM crosstalk are generated not only next to the pump but also close to the WDM signals. From Eq. (1) we can distinguish two extreme cases which depend on the WDM system allocation from the pump frequency $(\Delta \Omega)$ : either $\Delta \Omega$ is a multiple of $\Delta \omega$ or an odd multiple of half of $\Delta \omega$. When $\Delta \Omega=m \Delta \omega$ with $m$ an integer, parasitic PC FWM frequencies are perfectly superimposed with input WDM channels and then induce maximum crosstalk. On the other hand, by choosing the pump-channel spacing as odd multiples of the channel spacing, that is, $\Delta \Omega=(2 m+1) / 2 \cdot \Delta \omega$, parasitic PC FWM frequencies are created in between the channels and do not interfere with them [8]. Therefore, for reducing the overall crosstalk in WDM-FOPA, a simple way consists in adjusting the pump-channel detuning to $(2 m+1) / 2 \cdot \Delta \omega$. Nonetheless, the overall crosstalk will not be totally suppressed because the CC-FWM still remains significant [9].

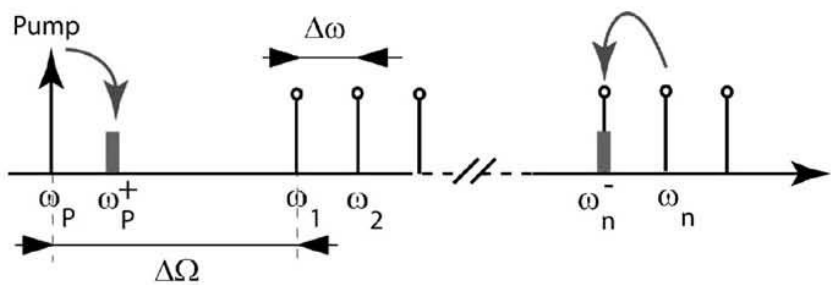

Fig. 1. Schematic of the pump channel FWM-induced crosstalk in single pump FOPA.

\section{Amplification of 64 NRZ Channels}

We investigated this PC crosstalk by using the Split-Step Fourier method to numerically integrate the following nonlinear Schrödinger equation which governs FOPA [10]:

$\frac{\partial A}{\partial z}+\frac{\mathrm{i}}{2} \beta_{2} \frac{\partial^{2} A}{\partial \tau^{2}}-\frac{1}{6} \beta_{3} \frac{\partial^{3} A}{\partial \tau^{3}}-\frac{\mathrm{i}}{24} \beta_{4} \frac{\partial^{4} A}{\partial \tau^{4}}=\mathrm{i} \gamma|A|^{2} A$

where $A(z, \tau)$ is the electric field amplitude, $\tau$ the time expressed in a reference frame moving at the group velocity of the light, $\beta_{m}(m=2, \ldots, 4)$ the $m$ th dispersion coefficients and $\gamma$ the nonlinear coefficient. We used typical FOPA parameters based on highly nonlinear dispersionshifted fibers: $\beta_{2}\left(\omega_{\mathrm{P}}\right)=0 \mathrm{~s}^{2} \mathrm{~m}^{-1}, \beta_{3}=0.6 \times 10^{-40} \mathrm{~s}^{3} \mathrm{~m}^{-1}$ $\left(D_{\mathrm{S}}=0.037 \mathrm{ps} \mathrm{nm}^{-2} \mathrm{~km}^{-1}\right), \quad \beta_{4}=-2.85 \times 10^{-55} \mathrm{~s}^{4} \mathrm{~m}^{-1}$, $\gamma=18 \mathrm{~W}^{-1} \mathrm{~km}^{-1}, L=300 \mathrm{~m}, \lambda_{\mathrm{P}}=1550 \mathrm{~nm}$ and $P=$ $500 \mathrm{~mW}$. We consider the amplification of 64 non-return to zero (NRZ) data modulation format at a bit rate of $R=10 \mathrm{Gbits} / \mathrm{s}$, with $50 \mathrm{GHz}$ channel spacing $(\Delta \omega)$ and $-20 \mathrm{dBm}$ mean power per channel. The NRZ data streams of each channel are independent and randomly generated. The cw pump is phase modulated at $3 \mathrm{GHz}$ with a PRBS sequence with rise time and fall time of $90 \mathrm{ps}$ in order to get a realistic configuration. With these typical parameters, the gain bandwidth is about $40 \mathrm{~nm}$ and the maximum gain reaches $17 \mathrm{~dB}$, as shown in Fig. 2. Fig. 3a and b represent a zoom on the input (grey) and output (black) WDM signals spectrum. As it can be seen, we clearly see parasitic waves resulting from both $\mathrm{PC}$ and CC FWM processes on the output spectrum. Fig. 3a corresponds to a multiple of $\Delta \Omega=0.75 \mathrm{THz}$ (i.e., $15 \times 50 \mathrm{GHz}$ ) and, as explained previously, we see that parasitic waves nearby WDM channels (black arrows) are spectrally shifted from the first channel by $m \Delta \omega$. They originate from both PC FWM and CC FWM. They clearly appear in between the first WDM channel and the pump on the output spectrum but they are also generated onto the WDM channels, which suffers from both crosstalk sources. On the other hand, we show in Fig. 3b the case of an odd multiple of half of $\Delta \Omega=0.775 \mathrm{THz}$ (i.e., $15.5 \times 50 \mathrm{GHz}$ ). The parasitic waves

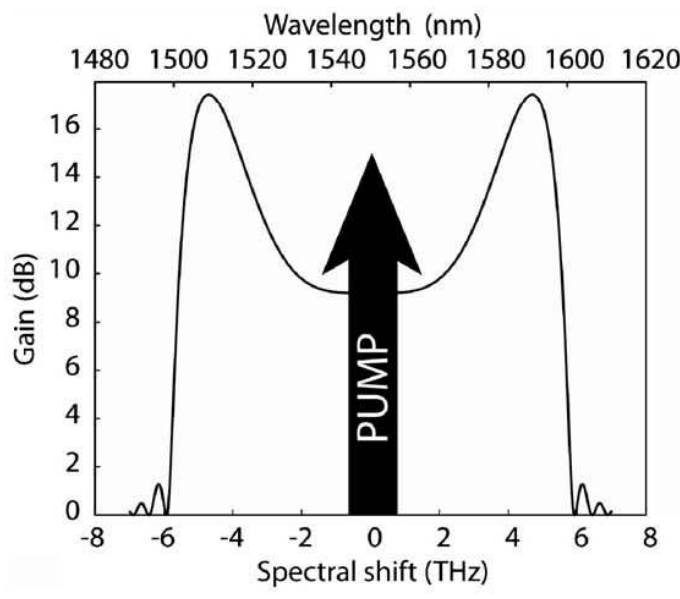

Fig. 2. Parametric gain band. 

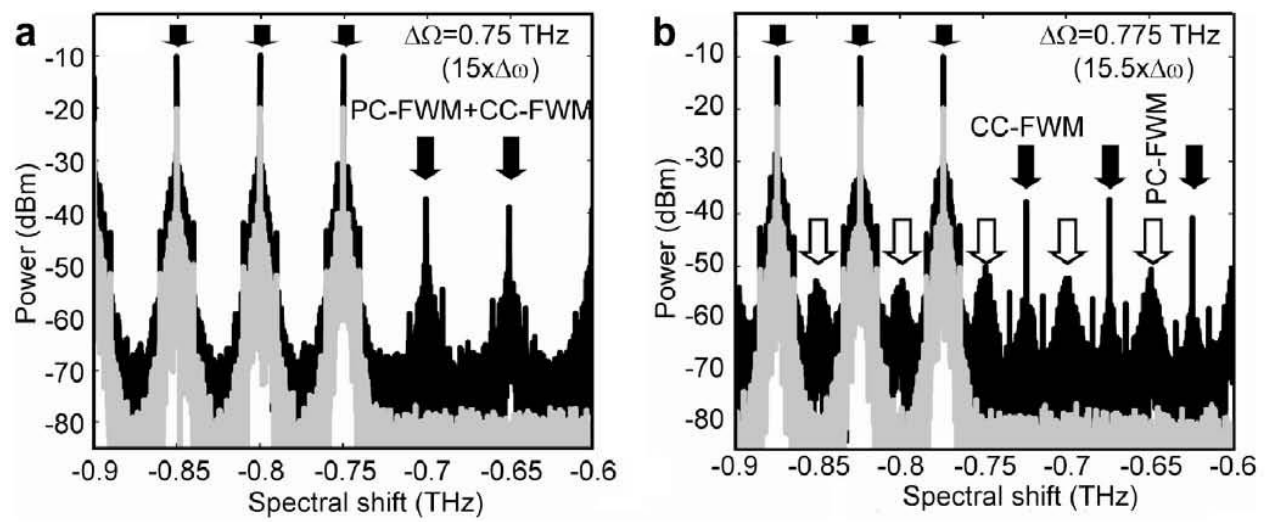

Fig. 3. Zoom on the input (grey) and output (black) spectrum of 64 NRZ signals at 10 Gbits/s: (a) for $\Delta \Omega=15 \times \Delta \omega$ and (b) for $\Delta \Omega=15.5 \times \Delta \omega$.

induced by PC FWM are now shifted by $(2 m+1) / 2 \cdot \Delta \omega$ from the first channel (white arrows) and thus, fall in between two channels. In this case the crosstalk, i.e., parasitic waves generated on the input channel, is reduced to the CC FWM process only (black arrows). Some spurious harmonics are still visible in the spectral range bounded by the first WDM channel and the pump [4]. Hence, by simply looking at the output spectrum we see that parasitic waves originating from PC FWM can either be generated onto the WDM channels (Fig. 3a) leading to maximum crosstalk, or just in between (Fig. 3b). In this latter case, they cannot interfere with the WDM channels and therefore, crosstalk must be lower. Moreover, total suppression of these parasitic FWM sidebands can be achieved by using a Fabry-Pérot filter that matches the WDM channel spacing, as suggested in Ref. [8]. To quantify the crosstalk, we measured the eye diagram aperture of each channel versus $\Delta \Omega$. We used the $Q^{\prime}$ quality factor as a criterion [11]. Eye diagrams were obtained by modeling a realistic receiver as in Ref. [12] and we naturally take $Q^{\prime}=1$ for a perfect eye opening as a reference. Then, the overall crosstalk for the 64 WDM amplified system is assessed by averaging the $64 Q^{\prime}$ values $\left(\overline{Q^{\prime}}\right)$. We performed numerical simulations for pump-channel spacing $\Delta \Omega$ ranging from $0.75 \mathrm{THz}$ to $1 \mathrm{THz}$ with $5 \mathrm{GHz}$ steps and for WDM signals located either on the Stokes or on the anti-Stokes side of the pump. Each simulation is performed with WDM channels carrying a random NRZ data stream. The results of our numerical simulations are illustrated in Fig. 4. As it can be seen, $\overline{Q^{\prime}}$ evolves in a sinusoidal way with a $50 \mathrm{GHz}$ (i.e., $\Delta \omega$ ) period, with maximum values of $\overline{Q^{\prime}}$ (best eye diagram) all located around $\Delta \Omega=(2 m+1) / 2 \cdot \Delta \omega$ (vertical dotted lines) and minimum ones located around $\Delta \Omega=m \Delta \omega$. Similar qualitative behavior is observed whenever the WDM signals are launched on the Stokes or anti-Stokes sides of the pump. Our results confirm that the pump-channel spacing $\Delta \Omega$ has indeed a strong impact on the overall crosstalk of WDM signals. It is worth noting that the improvement obtained by choosing the best pump-channel spacing depends on the input power signals. Indeed, beating process is more efficient as the waves become stronger, so the improvement increases with input signal power and/ or FOPA gain. Therefore, choosing the pump-channel spacing as $\Delta \Omega=(2 m+1) / 2 \cdot \Delta \omega$ is a useful tool to minimize the crosstalk induced by PC FWM.

In addition, Fig. 4 shows that $\bar{Q}$ is always larger for WDM signals located on the Stokes side rather than on the anti-Stokes side of the FOPA spectrum. First, this asymmetry cannot be attribute to CC-FWM since the absolute dispersion value is the same on both sides of the FOPA spectrum $\left(\beta_{2}\left(\omega_{\mathrm{P}}\right)=0\right)$. It could be intuitively due to stimulated Raman scattering but we do not include it in our simulations. Actually, this asymmetry also results from the PC crosstalk. To get better insight, phase-matching between the four waves of PC-FWM process must be considered. It can be expressed as [13]:

$\kappa=\beta_{2}\left(\omega_{\mathrm{C}}\right) \times\left(\Delta \omega_{\mathrm{S}}^{2}-\Delta \omega_{\mathrm{p}}^{2}\right)+\gamma P_{0}$

where $\omega_{\mathrm{C}}$ is the central frequency between the pump and the $n$th channel, $\beta_{2}\left(\omega_{\mathrm{C}}\right)$ is the value of second order disper-

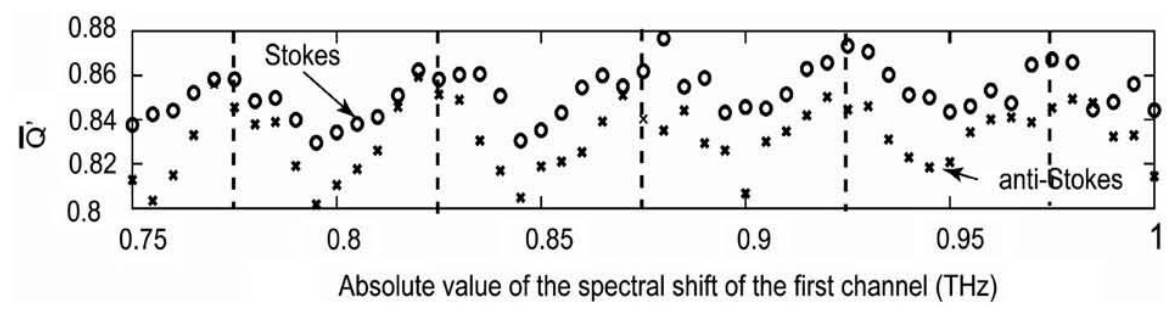

Fig. 4. $\overline{\Delta Q^{\prime}}$ versus the pump channel spacing $\Delta \Omega$, for 64 WDM signals located on the Stokes (circles) and anti-Stokes (crosses) side of the FOPA spectrum, respectively. Dotted lines correspond to $\Delta \Omega=(2 m+1) / 2 \cdot \Delta \omega$. 


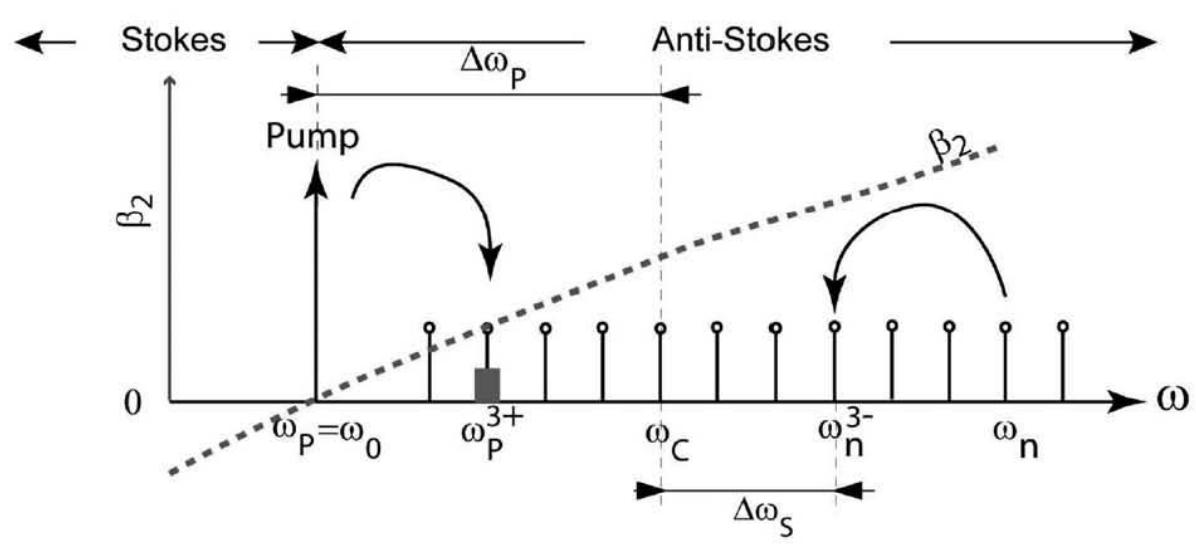

Fig. 5. Scheme illustrating the phase-matched pump channel FWM crosstalk on the anti-Stokes side of FOPA. Second-order dispersion is plotted in dashed gray line.

sion at $\omega_{\mathrm{C}}, \Delta \omega_{\mathrm{S}}$ is the spectral detuning between the $n$th channel and the central frequency, $\Delta \omega_{\mathrm{P}}$ is the one between the pump and $\omega_{\mathrm{C}}$, and $P_{0}$ is the sum of the pump power and of the $n$th channel power. Let us now consider as an example the non-degenerate FWM process depicted in Fig. 5 where interaction of the pump, the $n$th and the $(n-3)$ th channel generates the $\omega_{\mathrm{p}}^{3+}$ sideband for $\Delta \Omega=m \Delta \omega$. From Eq. (3), we deduce that phase-matched interactions $(\kappa=0)$ which correspond to the strongest crosstalk can occur when $\beta_{2}\left(\omega_{\mathrm{C}}\right) \times\left(\Delta \omega_{\mathrm{S}}^{2}-\Delta \omega_{\mathrm{p}}^{2}\right)<0$. If $\beta_{2}\left(\omega_{\mathrm{C}}\right)>0$ (case illustrated in Fig. 5, i.e., when signals are launched on the anti-Stokes side of the pump), perfect phase matching is thus fulfilled when $\left(\Delta \omega_{\mathrm{S}}^{2}-\Delta \omega_{\mathrm{p}}^{2}\right)<0$. We see in Fig. 5 that it involves WDM channels located in between the pump and the $n$th channel. On the other hand, if $\beta_{2}\left(\omega_{\mathrm{C}}\right)<0$, i.e., for signals on the Stokes side, perfect phase-matching is achieved for waves located outside the spectral region bounded by the pump and the $n$th channel. However, in this latter case, perfect phase-matched spectral bands are very narrow [13]. We can deduce that the impact of this PC FWM process is much less efficient in comparison with the case where $\beta_{2}\left(\omega_{\mathrm{C}}\right)>0$. Consequently PC FWM is enhanced when the WDM signals are launched on the anti-Stokes side of the pump. As an example, we plotted in Fig. 6 the phase mismatch $\kappa$ between the pump and the 64th channel for both the anti-Stokes (solid line) and the Stokes sides (dashed line) of the FOPA. As it can be seen, phase-matching can be satisfied for the anti-stokes side only of the FOPA spectrum.

Note that we did not develop a full analytical study of the whole FWM interactions because it involves 65 coupled equations. Nevertheless, our simple considerations allow us to clearly explain the numerical results presented on Figs. 3 and 4 .

\section{Discussion}

We must stress that our conclusions go beyond the FOPA configuration under study. In most FOPAs, the wavelength spacing between the pump and the zero disper-

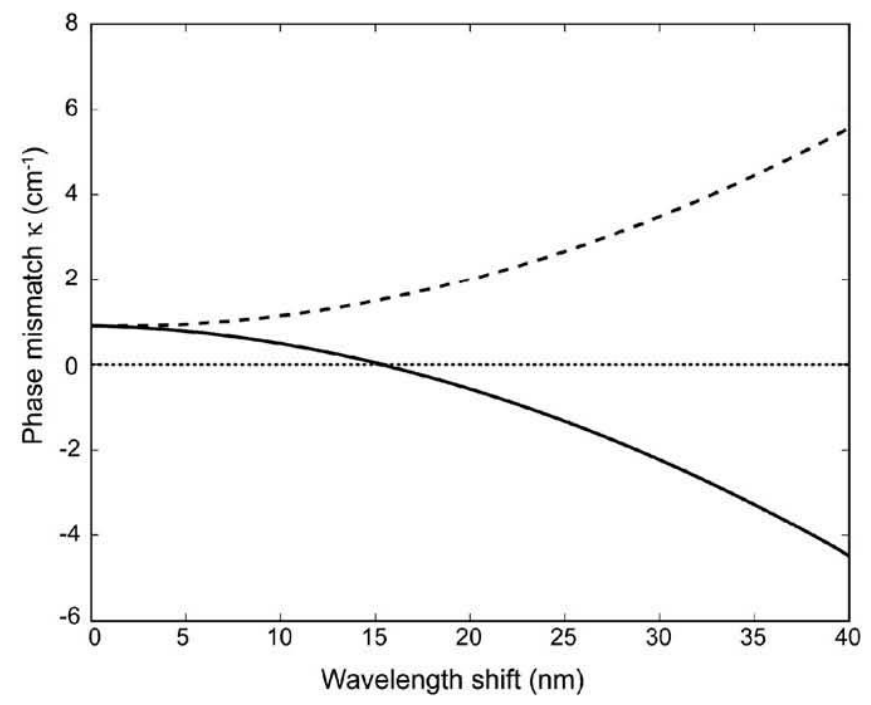

Fig. 6. Phase mismatch $\kappa$ of Eq. (3) between the pump and the 64th channel for both the Stokes (dashed line) and the anti-Stokes (solid line) sides of the FOPA spectrum.

sion wavelength of the fiber is of the order of a few tenths of nanometers (i.e., a few tens of Gigahertz) [1-4]. This value is negligible in comparison with the spectral shift between the pump and the first WDM channel. Therefore, the group-velocity dispersion undergone by the WDM channels is always positive (negative) for signals launched on the anti-Stokes (Stokes) side of the FOPA. PC-FWM phase-matched processes (Eq. (2)) depend on the groupvelocity dispersion value at $\omega_{\mathrm{C}}$. Therefore, similar phasematched PC-FWM processes are expected to appear in typical FOPA configurations. Lower crosstalk values for signals launched on the Stokes side of the pump are thus, a general behaviour of single-pump FOPAs.

Though a study of FOPAs driven by two-pump waves is out of the scope of this paper, we can infer from the present study that the first and the last WDM signal must be also located at $\Delta \Omega=(2 m+1) \times \Delta \omega / 2$ from each pump, respectively. Indeed similar pump-channel interactions take place with each pump. 


\section{Conclusions}

In this paper we have investigated through simple considerations and numerical simulations the crosstalk due to four-wave mixing between equally-spaced WDM signals in single-pump FOPAs. We have numerically demonstrated that crosstalk can be reduced by properly tuning the WDM signal allocation with respect to the pump wavelength. We have also shown that crosstalk is also reduced when signals are located on the Stokes side of the pump frequency because of the absence of phase-matching. We believe that our conclusions can be useful as overall rules for designing future high-performance fiber optical parametric amplifiers in view of WDM applications.

\section{References}

[1] K. Krastev, J. Rothman, Crosstalk in a fiber parametric amplifier, in: Eur. Conf. Optical Communication (ECOC 2001), vol. 1, Paper WeL3.5, p. 378
[2] T. Torounidis, H. Sunnerud, P.O. Hedekvist, P.A. Andrekson, IEEE Photon. Technol. Lett. 15 (2003) 1061.

[3] J.L. Blows, Opt. Commun. 236 (2004) 115.

[4] J.M. Chavez Boggio, F.A. Callegari, J.D. Marconi, A. Guimaraes, H. Fragnito, Opt. Commun. 242 (2004) 471.

[5] J.M. Chavez Boggio, J.D. Marconi, H. Fragnito, Opt. Commun. 259 (2006) 94.

[6] F.A. Callegari, J.M. Chavez Boggio, H.L. Fragnito, IEEE Photon. Technol. Lett. 16 (2004) 434.

[7] J.L. Blows, P.F. Hu, J. Opt. Soc. Am. B 21 (2004) 989.

[8] M.N. Islam, O. Boyarz, IEEE J. Sel. Topics Quantum Electron. 8 (2002) 527.

[9] R.W. Tkach, A.R. Chraplyvy, F. Forghieri, A.H. Gnauck, R.M. Derosier, J. Lightwave Technol. 13 (1995) 841.

[10] G.P. Agrawal, Nonlinear Fiber Optics, Academic Press, San Diego, 2001.

[11] D. Penninkx, O. Audouin, Optically preamplified systems: Defining a new eye aperture, in: Optical Fiber Communication Conference/OFC'1998, Paper WM36, (February 1998, San Jose, California).

[12] F. Matera, M. Settembre, J. Lightwave Technol. 14 (1996) 1.

[13] C.J. McKinstrie, S. Radic, A.R. Chraplyvy, IEEE J. Sel. Topics Quantum Electron. 8 (2002) 538. 